\title{
Quantitative digital in situ senescence-associated $\beta$-galactosidase assay
}

\author{
Liran I Shlush ${ }^{1,2,3}$, Shalev Itzkovitz ${ }^{4}$, Ariel Cohen ${ }^{4}$, Aviad Rutenberg ${ }^{1}$, Ron Berkovitz ${ }^{1,2}$, Shiran Yehezkel ${ }^{1}$,
} Hofit Shahar ${ }^{1,2}$, Sara Selig ${ }^{1,3^{*}}$ and Karl Skorecki,

\begin{abstract}
Background: Cellular senescence plays important roles in the aging process of complex organisms, in tumor suppression and in response to stress. Several markers can be used to identify senescent cells, of which the most widely used is the senescence-associated $\beta$-galactosidase (SABG) activity. The main advantage of SABG activity over other markers is the simplicity of the detection assay and the capacity to identify in situ a senescent cell in a heterogeneous cell population. Several approaches have been introduced to render the SABG assay quantitative. However none of these approaches to date has proven particularly amenable to quantitative analysis of SABG activity in situ. Furthermore the role of cellular senescence (CS) in vivo remains unclear mainly due to the ambiguity of current cellular markers in identifying CS of individual cells in tissues.

Results: In the current study we applied a digital image analysis technique to the staining generated using the original SABG assay, and demonstrate that this analysis is highly reproducible and sensitive to subtle differences in staining intensities resulting from diverse cellular senescence pathways in culture. We have further validated our method on mouse kidney samples with and without diabetes mellitus, and show that a more accurate quantitative SABG activity with a wider range of values can be achieved at a pH lower than that used in the conventional SABG assay.

Conclusions: We conclude that quantitative in situ SABG assay, is feasible and reproducible and that the $\mathrm{pH}$ at which the reaction is performed should be tailored and chosen, depending on the research question and experimental system of interest.
\end{abstract}

\section{Background}

Cellular senescence (CS) is a term used to describe the process wherein somatic cells of complex eukaryotic organisms progressively lose replicative capacity. The relationship between CS and organismal aging is still unclear although recent studies in non-human and human primates have strongly implicated a correlation between organismal and cellular aging [1,2]. Overall, recent studies have suggested that CS is a final common pathway resulting from activation of the cellular DNA damage response (DDR) by various stressors that converge on the p53 and/or pRB pathways. Different DDR inducing stimuli can lead to various types of CS. Among those most thoroughly investigated is the activation of

\footnotetext{
* Correspondence: seligs@tx.technion.ac.il

'Laboratory of Molecular Medicine, Rappaport Faculty of Medicine and Research Institute, Technion, Haifa 31096, Israel

Full list of author information is available at the end of the article
}

DDR by telomere attrition which leads to cell cycle arrest termed replicative senescence (RS) or telomereinitiated CS [3-5]. Other well studied forms of CS include oncogene-induced senescence [6-8], cell structure induced senescence related to dysfunctional Lamin A [9], and stress-induced premature senescence (SIPS), the latter most thoroughly studied in relation to oxidative stress [10-12]. These various triggers of CS might not necessarily be mutually exclusive. Furthermore, DDR might not be the exclusive mechanism for triggering CS as protein damage, epigenetic changes [13] and additional processes have also been implicated [5,14]. In complex long-lived organisms CS is considered to be a tumor suppressor mechanism similar to apoptosis and autophagy [15]. However, in contrast to apoptosis and autophagy, which are irreversible and lead to cell death, senescent cells maintain partial metabolic functionality
C Biomed Central 
without dividing, and have been shown to have the capacity to revert back to a proliferative state [14].

Several markers of senescence have been described [5]. Among others these include G1 cell cycle arrest detected by lack of DNA replication, cytological markers such as senescence-associated heterochromatin foci (SAHF), senescence-associated DNA-damage foci, as well as cell structure changes such as cell size and lysosomal $\beta$-galactosidase activity detected at $\mathrm{pH} 6.0$ defined as senescence-associated $\beta$-galactosidase (SABG) activity $[16,17]$. Since first reported, SABG activity has been the most extensively utilized biomarker for CS both in in situ [16,18-20] and in in vitro studies (reviewed in [17]). In many studies the identification of cells as being senescent rests solely on the SABG assay. The popularity of this method can be attributed to its simplicity and apparent specificity for CS regardless of the initiating trigger, as well as the ability to visualize senescent cells in a heterogeneous population [17].

Despite the extensive utilization of the SABG assay for CS determination, the origin of SABG activity and its role in CS were unknown for several years following its initial description. A number of studies have proposed that lysosomal $\beta$-galactosidase activity increases in senescent cell up to a degree that surpasses a threshold level that renders the activity detectable at a suboptimal pH 6.0 [21,22]. A later study clearly demonstrated that the SABG activity arises from the lysosomal $\beta$-galactosidase 1 (GLB1) gene product [23]. In senescence cells, both the mRNA and the protein levels of this gene are significantly elevated, and the enzymatic activity increases concomitantly [23]. Furthermore, the enhanced enzymatic activity in senescence can be measured both at the optimal $\mathrm{pH}$ for activity - $\mathrm{pH} 4.5$ as well as at the suboptimal $\mathrm{pH}$ 6.0. These findings demonstrate that the significantly increased SABG activity at senescence is the basis for the activity detected at the suboptimal $\mathrm{pH}$ 6.0, and therefore used as a marker for senescence [23].

The extent of the senescence-induced increase in lysosomal $\beta$-galactosidase can be measured by Western blotting or soluble enzymatic activity [23], however the activity of $\beta$-galactosidase is most easily and robustly detected by histochemical staining with X-gal serving as a substrate [23]. In addition to age related accumulation of lysosomal $\beta$ galactosidase in senescent cells probably due to the increased lysosomal content in the cell, other yet unknown factors such as functional differences in senescent lysosomes may contribute to the very high levels of $\beta$-galactosidase observed by SABG staining at $\mathrm{pH} 6.0$ [23].

Despite its ease and utility, concerns have arisen regarding the specificity and reproducibility of the SABG assay. Studies that question the specificity of the SABG assay as a CS marker have found SABG activity in quiescent cell cultures [24], confluent non-transformed fibroblasts cultures $[25,26]$ and in serum starved cells [26]. Other concerns relate to the nature of the semi-quantitative measure. Previously described scoring has been based on experimentalist-dependent determination of a given cell as being positively or negatively stained, and may not be anchored in reproducible criteria. Furthermore, the intensity of blue staining in positive cells may be difficult to quantify, such that cells with strong, moderate, or weak blue staining may all be recognized as equally positive. This renders the method insensitive to subtle effects of various stressors on CS, and might contribute to the inconsistency in replicating SABG assay results in skin biopsies [25].

The foregoing motivated us to develop a quantitative in situ SABG assay, which could be more easily applicable and reproducible in the study of CS both in vitro and in situ. We have utilized the framework of the widely utilized protocol of the in situ SABG assay [16], and applied digital-image processing in order to perform quantification of the assay staining. In addition, we have also varied the $\mathrm{pH}$ of the assay to broaden the range of histochemically detectable activity. We show validation of this quantitative in situ SABG assay on cultured human foreskin fibroblasts and frozen kidney biopsies from normal and diabetic mice, stained under different assay conditions. The values derived from this analysis are termed $\beta$-galactosidase activity values (BGAVs) and are highly sensitive and reproducible.

\section{Results}

\section{Image processing of the standard SABG assay}

The SABG assay produces blue-green staining in the cytoplasm of positive cells, albeit there is great variability in the degree of staining among the positive cells. In order to objectively quantify this staining we utilized the protocol for SABG staining as previously described [16], and proceeded to capture and digitally analyze images of the stained cells. This quantification method, based on color intensity analysis of the photographed images, generates values of Cell Staining Intensity (CSI) measured in arbitrary intensity units. CSI values are the product of the ratio between the green plus blue values of each pixel in the manually marked cell cytoplasm and the total color (red, green and blue) in the same cytoplasm, divided by the cell surface area. Standardization of each single CSI value is achieved by dividing the CSI value with the adjacent background staining intensity (BSI) value (Figure 1A, B). We found that the background is uneven over the photographed area and therefore standardization to the figure background is best achieved by marking a background area adjacent to each analyzed cell. In order to decrease the signal to noise ratio, we applied a logarithmic transformation to the 


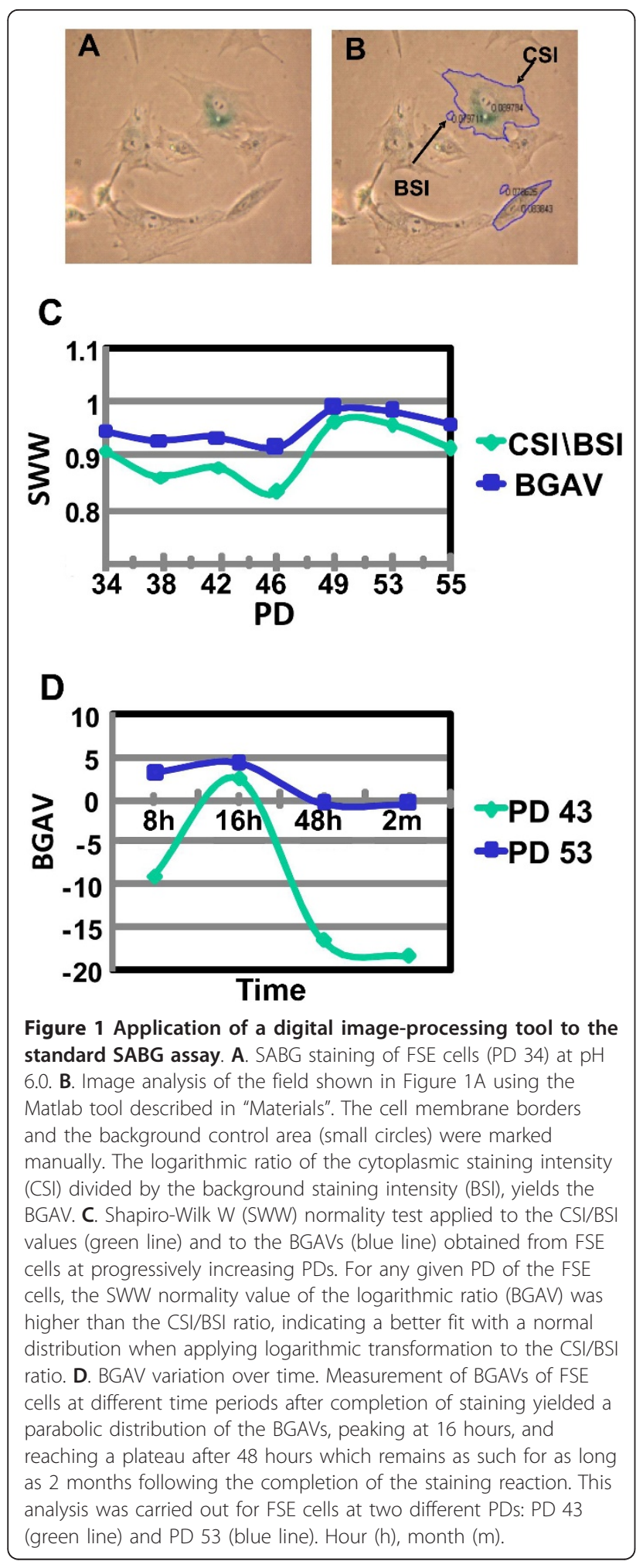

ratio CSI/BSI and termed the value obtained: BGAV. For each experiment a minimum of 250 cells were analyzed to generate BGAVs, which were further analyzed as mean values and distributions.
To apply this analysis to primary human fibroblasts aged in culture and in order to determine the preferred analysis strategy, we stained and calculated CSI/BSI and BGAVs for two different primary fibroblast cell lines, FSE and BJ, at various population doublings (PDs). We applied normality tests for these values, and found higher normal distribution indices for BGAVs versus CSI/BSI (Figure 1C). The Shapiro-Wilk W (SWW) BGAVs of 250 FSE cells at PD 38 was higher than the CSI/BSI ratio of the same cells, yielding a better fit to a normal distribution. Nevertheless, in most instances the BGAVs did not fit a normal distribution according to either Shapiro-Wilk W or Kolmogorov-Smirnov normality tests, and therefore in our statistical analysis we used the Kolmogorov Smirnov test, rather than the Student's t-test, which assumes normal distribution.

The first scoring of BGAVs was performed on images captured 8 hours following the start of the incubation with the X-Gal staining solution, immediately after stopping the staining reaction by washings with PBS, as described in "Methods". In order to examine whether the BGAVs change as a function of the time elapsed since the staining was completed, we continued to measure the mean BGAV of 250 cells at different time points following staining. We observed a parabolic behavior of the mean BGAV over time, peaking at 16 hours after the termination of the staining reaction (Figure 1D). The BGAVs reached a plateau 40 hours after the staining reaction was terminated and remained stable for as long as two months. We have chosen the values attained at the plateau stage as more reliable and stable markers for quantifying the degree of SABG staining and proceeded to acquire all our images at least 40 hours after completion of staining.

\section{BGAVs in Cellular Senescence pathways}

The SABG assay was originally introduced to measure RS [16]. In order to confirm that our quantification analysis indeed detects this type of senescence, we measured the mean BGAV in FSE and BJ fibroblasts at progressively increasing PDs. Our measurements in the FSE cells demonstrated a steady increase in BGAVs between PDs 34-58, and in BJ fibroblasts between PDs 57-65 (Figure 2). Analysis of cell surface areas from FSE cells at PDs 34 to 56 presented a concomitant gradual increase in the mean cell surface area from 24520 pixels to 39125 pixels respectively as expected in cells approaching CS, and this change was statistically significant $\left(\mathrm{p}<10^{-5}\right)$. We fully appreciate the fact that accurate cell area can only be calculated using confocal microscopy, though for the purpose of validating the quantitative SABG assay we did not consider this to be necessary. The increase in mean cell surface area would be expected to yield a lower mean BGAV in cells 


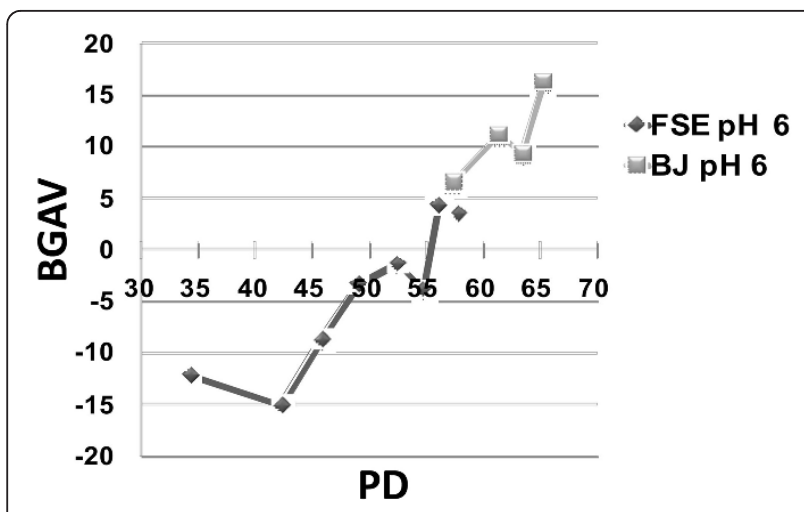

Figure 2 BGAVs change with increasing population doublings. The BGAVs obtained at pH 6.0 at progressively increasing PDs of both FSE and BJ fibroblast cells. FSE cells were analyzed between PD 34-58 and BJ cells were analyzed between PD 57-65. Both cell types were stained for SABG activity at weekly intervals.

approaching CS, since cell surface is the denominator for CSI. However, as demonstrated in Figure 2, the increase in the mean BGAV from $-12.2 \pm 13.7$ at PD 34 to $4.3 \pm 14.7$ at PD $56\left(\mathrm{p}<10^{-5}\right)$, validates the efficacy and reliability of this method to observe and measure changes in SABG staining associated with RS.

Many studies have shown that SIPS is also associated with positive SABG staining [12,27]. In order to further validate the reliability of our method, we exposed FSE cells at PD 34 to gradually increasing concentrations of $\mathrm{H}_{2} \mathrm{O}_{2}$ in order to induce acute SIPS. Indeed, an $\mathrm{H}_{2} \mathrm{O}_{2}$ concentration dependent increase in the mean BGAV was observed (Figure 3). The mean BGAV was significantly higher after exposure to $15 \mathrm{uM} \mathrm{H}_{2} \mathrm{O}_{2}(-3.4 \pm$ 17.8) in comparison to no added $\mathrm{H}_{2} \mathrm{O}_{2}(-12.2 \pm 13.7)$ $\left(\mathrm{p}<10^{-5}\right)$. No significant differences in BGAVs were observed between $\mathrm{H}_{2} \mathrm{O}_{2}$ concentrations of $15 \mathrm{uM}$ and $20 \mathrm{uM}(\mathrm{p}=0.2183)$. BGAVs reached a plateau at $25 \mathrm{uM}$

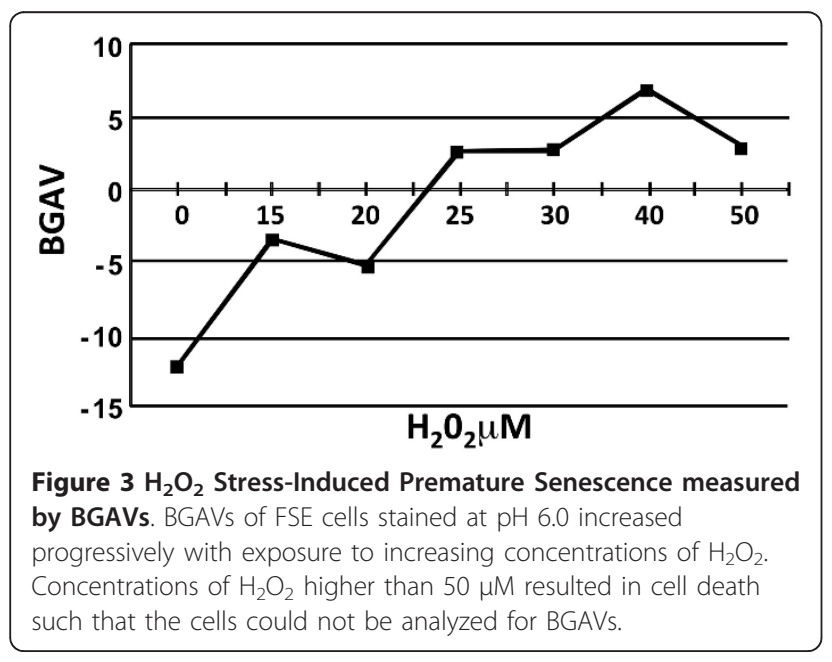

$\mathrm{H}_{2} \mathrm{O}_{2}$. Cell death occurred when cells were exposed to $\mathrm{H}_{2} \mathrm{O}_{2}$ concentrations higher than $50 \mathrm{uM}$ such that the cells could not be analyzed for BGAVs at these concentrations. Hence, BGAVs appear useful in documenting SIPS-induced SABG staining, as well.

\section{The effect of $\mathrm{pH}$ on BGAVs}

Recent studies have demonstrated that SABG activity at pH 6.0 in mammalian cells emanates from the activity of the lysosomal enzyme GLB1 [23]. This $\mathrm{pH}$ is suboptimal for this enzyme's activity that normally is carried out in the highly acidified lysosomes. The optimal $\mathrm{pH}$ for lysosomal $\beta$-galactosidase activity in human fibroblasts and leukocytes is situated between $\mathrm{pH} 4.2-4.6$, and activity declines by approximately $25 \%$ at $\mathrm{pH} 5.0$ and by $50 \%$ at pH 5.5 [28]. Apparently, only senescent cells with high $\beta$-galactosidase activity yield detectable staining at $\mathrm{pH} 6.0$ [23]. Accordingly, it has been the accepted convention to date to carry out the SABG assay at $\mathrm{pH} 6.0$, since at $\mathrm{pH} 4.0$ most cells are positively stained due to basal lysosomal $\beta$ galactosidase activity (Figure 4A) and therefore differentiation between levels of activity was considered not feasible at $\mathrm{pH}$ 4.0. However the $\beta$-galactosidase activity measured in late- compared to early-passage fibroblasts was six to seven-fold higher both at pH 4.5 and $\mathrm{pH} 6.0$ [23]. We therefore proceeded to study whether our quantification method might be able to measure differences in staining
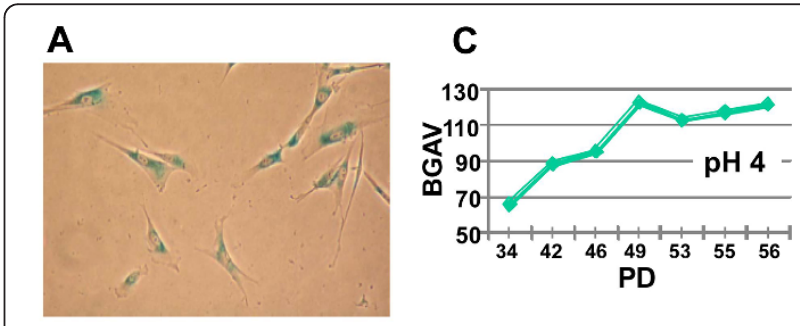

B

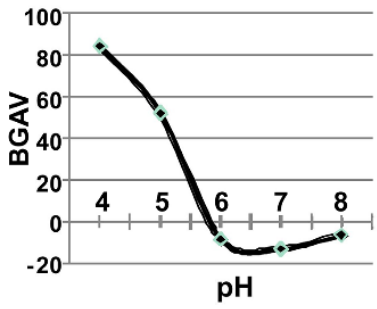

D

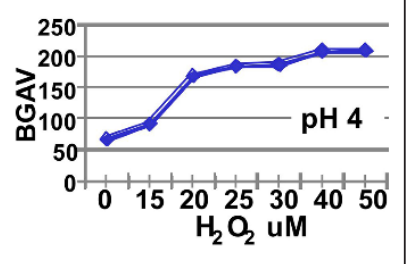

Figure $4 \mathbf{p H}$ effect on BGAVs. A. FSE cells at PD 34 stained at pH 4.0. All cells demonstrated some degree of staining. B. BGAVs are inversely related to the $\mathrm{pH}$ at which the reaction was carried out. Staining was performed on FSE cells at PD 42 under different pH conditions. $\mathbf{C}$. The effect of increasing PDs on BGAVs obtained at $\mathrm{pH}$ 4.0. FSE cells at progressively increasing PDs were stained at $\mathrm{pH} 4.0$ and analyzed for BGAVs. D. The effect of $\mathrm{H}_{2} \mathrm{O}_{2}$ on $\mathrm{BGAVs}$ obtained at $\mathrm{pH}$ 4.0. FSE cells were treated with progressively increasing $\mathrm{H}_{2} \mathrm{O}_{2}$ concentrations and BGAVs were determined at each concentration. 
intensities obtained at $\mathrm{pH}$ values other than $\mathrm{pH} 6.0$ in fibroblasts at early and late PDs. To this end, we first measured BGAVs obtained at different levels of $\mathrm{pH}$ in FSE cells at PD 39, a stage at which fibroblast cells are barely stained at $\mathrm{pH}$ 6.0. As shown in Figure 4B, the BGAVs decreased as the $\mathrm{pH}$ increased, and the highest BGAVs were attained when staining was performed at $\mathrm{pH}$ 4.0. Following this finding we analyzed the BGAVs obtained at $\mathrm{pH} 4.0$ during RS and SIPS. We observed that both at RS (Figure 4C) and SIPS (Figure 4D) staining at $\mathrm{pH} 4.0$ increased the resolution between the BGAVs at low PDs, or at low $\mathrm{H}_{2} \mathrm{O}_{2}$ values. While the mean $\mathrm{pH}$ 4.0-BGAV at PD $34(65.6 \pm 45.5)$, was significantly lower than at PD $42(88.4 \pm 60.4)(\mathrm{p}=0.0001)$ (Figure $4 \mathrm{C}$ ), this significant difference could not be documented at the same PDs with $\mathrm{pH}$ 6.0-staining (Figure 2). Similarly, readily discernible differences were obtained while comparing the mean BGAV at $15 \mathrm{uM}$ $\mathrm{H}_{2} \mathrm{O}_{2}$ compared with $20 \mathrm{uM} \mathrm{H} \mathrm{H}_{2} \mathrm{O}_{2}$ when staining was done at $\mathrm{pH} 4.0$, however this was not the case at $\mathrm{pH}$ 6.0. Therefore, we conclude that in order to measure smaller increments in the SABG activity or to perform this assay more accurately, it is beneficial to perform the staining procedure also at $\mathrm{pH} 4.0$ due to the increased sensitivity of the BGAVs at this $\mathrm{pH}$. However this requires a rigorous quantitative assay for histochemical staining intensity in order to distinguish the range of intensity of positive staining.

\section{The effect of cell density on BGAVs}

Earlier studies have reported that the degree of SABG staining is influenced by cell density, such that more intense staining is obtained when cells are closer to confluence [25]. In order to determine whether confluence effects are evident using our analytic approach, the mean BGAV was determined in wells at different confluence levels. ANOVA analysis of the mean BGAV at $\mathrm{pH} 4.0$ in two duplicate experiments using FSE cells showed no differences with varying cell density $(\mathrm{p}=$ 0.1 ), even when cells were plated at a high density (250,000 cells per well) (Figure 5).

\section{In situ SABG assay}

The potential roles CS plays in organismal aging and in tumor suppression raise the importance of a reliable quantitative method to determine CS in vivo. We have chosen to focus in the current study on kidney biopsies, in which CS has been described in various forms of kidney pathology [29-31]. Specifically, we have focused on type 2 diabetes mellitus (DM) wherein increased CS, quantified by SABG and p16INK4a, has been demonstrated in human subjects [32]. We have used a wellaccepted streptozotocin (STZ) model of murine diabetes mellitus [33] in order to analyze SABG activity in renal

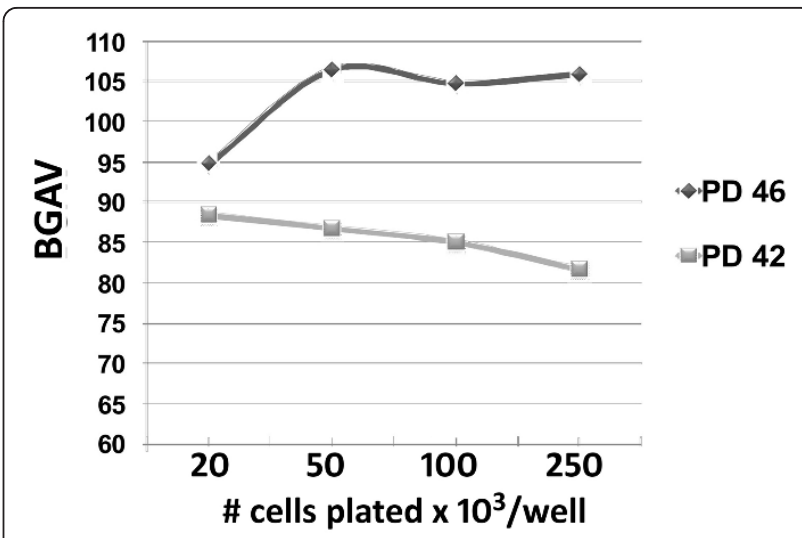

Figure 5 Cell Density and BGAVs. FSE cells at PDs 42 and 46 were plated at increasing densities (20,000 -250,000 cells/well) and stained at pH 4.0. BGAVs were determined at the different cell densities

tubules 7, 9 and 13 weeks following the administration of STZ. At pH 6.0 no positively stained kidney cells were detected and analysis by our software indicated that the mean BGAV was not statistically different between diabetics and controls at all 3 time points (Figure 6B). We hypothesized that $\beta$-galactosidase activity in mouse kidney cells may be low and detectable only at the optimal $\mathrm{pH}$, and therefore applied the SABG assay also at $\mathrm{pH}$ 4.0. Indeed, the BGAVs measured in tubular cells at $\mathrm{pH} 4.0$ differed significantly between the kidney samples of the diabetic mouse and the controls at all three time points ( 7 weeks $\mathrm{p}<0.004 ; 9$ weeks $\mathrm{p}<$ $0.0009 ; 13$ weeks $\mathrm{p}<10 \mathrm{E}^{-8}$ ) (Figure 6A). The staining was specific to tubular cells and no positive staining was detected in the glomeruli or interstitial tissue from both diabetic and control mice. In addition, the analysis revealed that the BGAVs at $\mathrm{pH} 4.0$ increased gradually in both diabetics and controls between week 7 and week $13\left(\mathrm{p}=5 \mathrm{E}^{-6}, 6 \mathrm{E}^{-6}\right.$ respectively) reflecting changes in SABG activity most likely due to aging.

In order to verify that the SABG staining indeed detects senescent cells in the kidney, we proceeded to analyze the kidney samples for an additional marker of senescence. We performed immunofluorescent (IF) staining with an antibody for $\gamma-\mathrm{H} 2 \mathrm{AX}$, a quantitative indicator of senescence both in vivo and in vitro [34]. One hundred nuclei in tubular cells of both diabetic and control mice at the 13-week time point were scored for the number of foci present in the nucleus (Figure 7). The number of nuclei containing one or more $\gamma$-H2AX foci was significantly higher in the tissue originating from the diabetic mouse in comparison to the control mouse (Pearson's chi-square $=44.9, \mathrm{p}=2.1 \mathrm{E}^{-11}$ ). Moreover, by comparing the percentage of $\gamma$-H2AX-positive nuclei with $\mathrm{pH}$ 4-SABG-positive tubules (defined as 


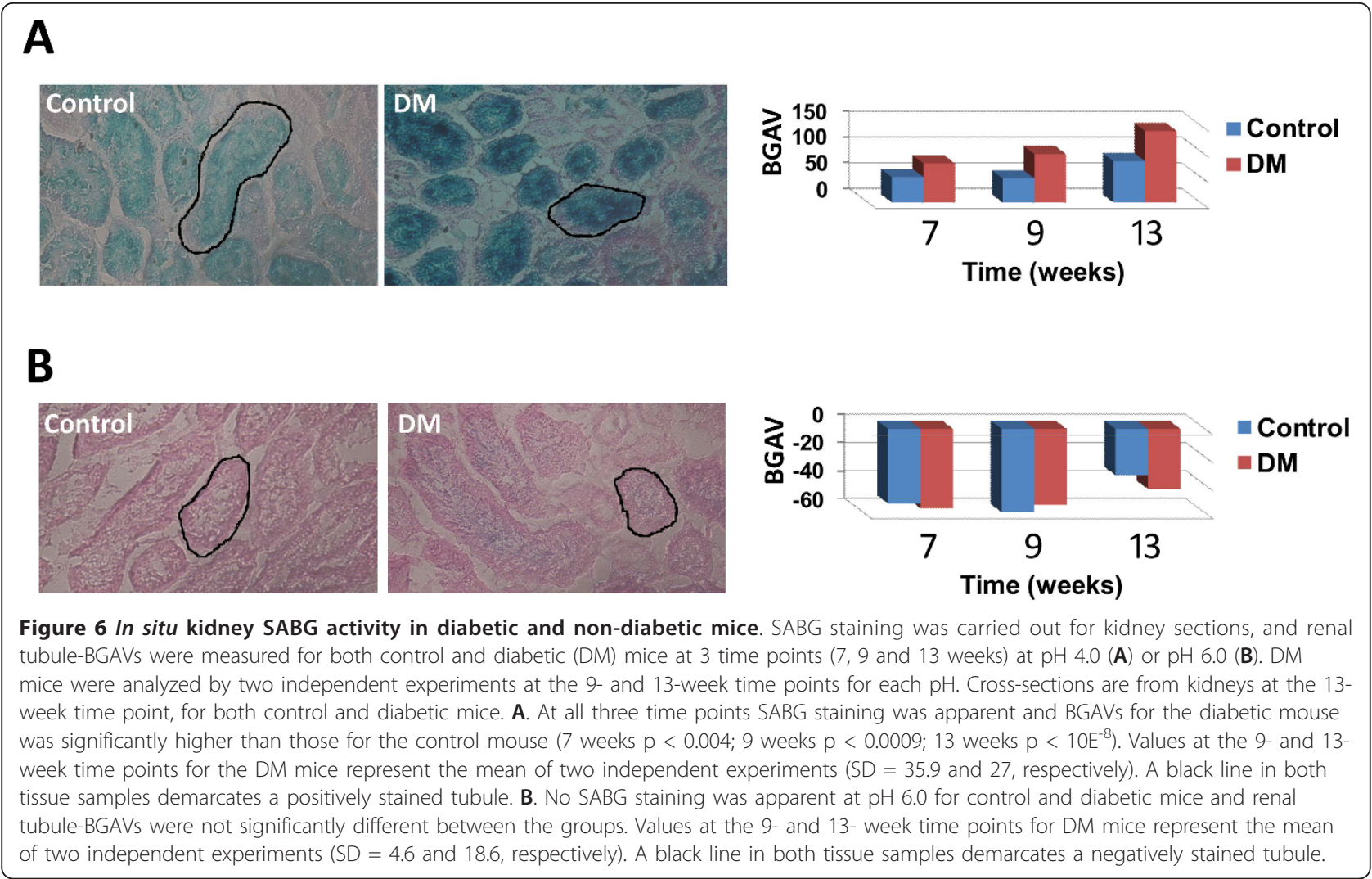

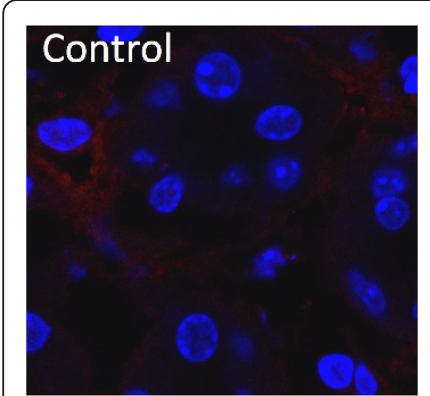
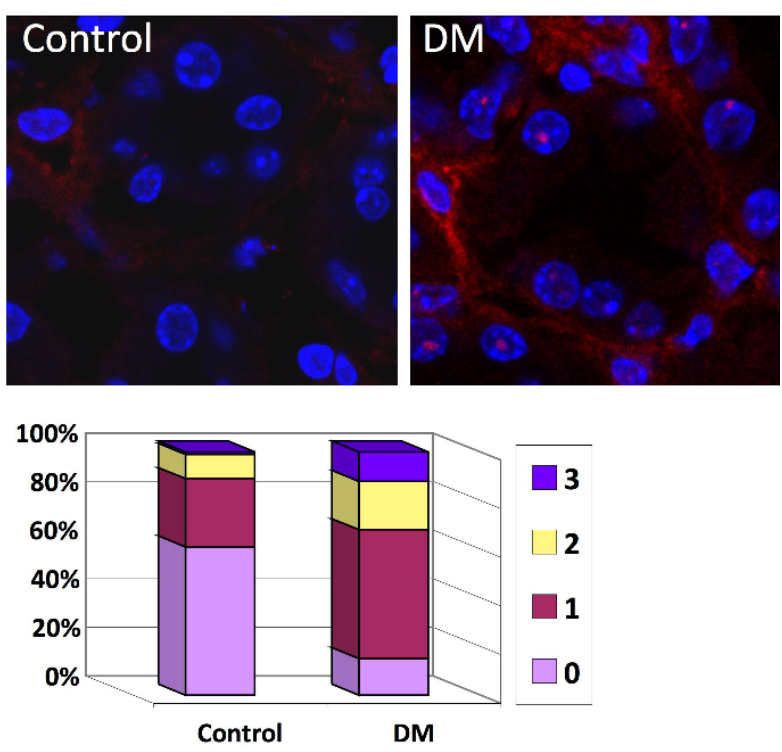

Figure $7 \gamma-\mathrm{H} 2 \mathrm{AX}$ foci in diabetic and non-diabetic mice. $\gamma-\mathrm{H} 2 \mathrm{AX}$ staining was carried out for kidney sections at the 13-week time point for both control and diabetic (DM) mice. Red - $\gamma-\mathrm{H} 2 \mathrm{AX}$ signals, Blue - DAPI nuclear staining. One hundred tubule cells from each sample were analyzed for the number of $\gamma$-H2AX foci present in the nucleus. The percentage of cells displaying 0-3 foci per nucleus appears in the graph ( 3 foci was the maximum number of foci detected per nucleus). demonstrating BGAV values greater than the average score for the corresponding control tubules), the correlation coefficient was calculated to be 0.96 , providing further reassurance that indeed the SABG staining at pH 4.0 identifies senescent cells.

\section{Discussion}

Cellular senescence is a final common pathway for a number of DDR pathways, and appears to play a major role in normal aging, acute stress conditions, and possibly as a tumor suppressor mechanism [5,14]. Despite its wide relevance to both normal tissue kinetics, and organismal aging, CS lacks definitive well-characterized and easily measured biomarkers. Senescence-associated $\beta$ galactosidase staining has been the most widely used technique to characterize senescent cells, however several recent studies highlight a number of caveats using this assay [25,26,35]. A major limitation of the SABG assay is the subjective assignment of cells as being positively or negatively stained. As a result, new quantitative SABG assays have been developed. These methods include measurement in cell extracts of the conversion rate of 4-methylumbelliferyl-D-galactopyranoside (MUG) to the fluorescent hydrolysis product 4-methylumbelliferone (4-MU) [36] and a method based on the levels of fluorescein di-( $\beta)$-D-galactopyranoside in the 
suspension buffer [37]. A quantitative chemiluminescent assay has also been recently reported [38], as well as FACS staining of SABG [39]. All these methods have yielded promising results for a quantitative measure of SABG, but are not designed for in situ quantification of SABG activity. We have used the platform of the original SABG assay [16] as the basis for developing an in situ reproducible quantitative assay. The digital imageanalysis we describe herein enables the quantification of SABG staining in uniform units, and distinguishes different degrees of staining. In addition, our analysis suggests that SABG staining is not influenced by cell density up to a certain degree of confluence in either pre-senescent or senescent cells (Figure 5).

A further modification and validation of this assay is varying the $\mathrm{pH}$ at which the assay is carried out to include $\mathrm{pH} 4.0$, at which biochemical activity of the GLB1 product is significantly higher [23]. The original description of SABG assay at pH 6.0 was meant to generate a threshold at which only cells with senescencedependent accumulation of the enzyme (now known to be the GLB1 gene product) and consequently higher activity stain positively. Qualitative SABG assay at a higher $\mathrm{pH}$ might be simpler and faster for interpretation when striking qualitative differences are being assessed. However, staining at $\mathrm{pH} 4.0$ renders the assay more sensitive to subtle changes, such as occur under conditions of metabolic stress, as demonstrated by the intensity of the staining after treatment with hydrogen peroxide. SABG staining at $\mathrm{pH} 4.0$ also enabled identification of CS in kidney samples of diabetic mice that was not evident at $\mathrm{pH}$ 6.0. While $\mathrm{pH} 4.0-4.5$ is the optimal $\mathrm{pH}$ for SABG activity, [36,40], without the digital analysis, subjective scoring will lose its utility for determination of CS as most cells will seem to be positively stained. Thus, in conclusion the $\mathrm{pH}$ of the assay should be adjusted in accordance with the biological question.

An evident advantage of a histochemical assay over a fluorimetric approach, as described by Yang and $\mathrm{Hu}$ [37], is the capacity for in situ quantification to shed light on CS at the level of individual cells and not only at the level of a whole cell population. Furthermore, the main innovation in our method is the ability to quantify in situ mixed cellular tissue sections and evaluate the SABG activity in specific cell types. Since many tissues are constituted of various types of cells, only the in situ staining can provide insights into the type of cells in a mixed population demonstrating high SABG activity.

As noted above, in situ visualization of SABG in diabetic mouse kidneys was possible only when the SABG assay was performed at $\mathrm{pH}$ 4.0. Previous SABG activity studies on in situ samples [16] have shown a qualitative increase in SABG activity in tissues from elderly patients even at $\mathrm{pH}$ 6.0. However no such staining was evident at
$\mathrm{pH} 6.0$ in the kidneys of the diabetic mice in the current study. Other studies in which SABG assay was used as a marker for CS in tissues have also frequently yielded conflicting results [25], and it has been suggested that activity is not causally related to CS induction [23]. In this study we have confirmed by an independent assay that indeed the kidneys of the diabetic mice harbor senescent cells. Herein we suggest that the ambiguity related to the SABG staining as a CS marker in tissues might have been the result of measurement of SABG activity at a high and suboptimal $\mathrm{pH}$. However, once again we emphasize that extending the assay to the more sensitive $\mathrm{pH}$ of 4.0 must be coupled with quantification of staining to achieve interpretable results.

\section{Conclusions}

Utilization of an objective quantitative measurement of SABG activity by digital image analysis has proved to be accurate and reproducible in detecting cellular senescence derived by several stimuli. The increased SABG activity observed among in situ tissue sections from diabetic mouse kidneys was observed only when the assay was performed at a physiologic $\mathrm{pH} 4.0$, and the analysis was enabled due to the use of computer aided image analysis. A major advantage and novelty of this approach in comparison to other qualitative and quantitative SABG methods is the ability to analyze tissue sections in the study of in situ senescence.

\section{Methods}

\section{Cell culture conditions}

Human primary foreskin fibroblasts (FSE) [41] and BJ [42] (obtained from Woodring Wright, University of Texas, Southwestern Medical Center, TX, USA) were cultured and brought to replicative senescence as described previously [41].

\section{$\mathrm{H}_{2} \mathrm{O}_{2}$ treatment of cells}

FSE cells were exposed to $\mathrm{H}_{2} \mathrm{O}_{2}$, diluted in PBS, at different concentrations $(0-100 \mu \mathrm{M})$. Following incubation with $\mathrm{H}_{2} \mathrm{O}_{2}$ cells were washed with PBS and grown for an additional 48 hours in culture medium prior to staining. Control cultures at the same population doubling followed the same schedule of medium changes without exposure to exogenous $\mathrm{H}_{2} \mathrm{O}_{2}$.

\section{Senescence associated $\beta$-galactosidase assay}

Cells were seeded 48 hours prior to staining at $2-4 \times$ $10^{4}$ cells/well in six well plates. This cell density ensures that the staining is performed before the cultures reach confluence. SA- $\beta$-Gal staining was performed as previously described with minor modifications [16]. Briefly, the cells were washed with cold PBS, and fixed for 5 min with $0.5 \%$ glutaraldehyde diluted in cold PBS. After 
fixation, cells were washed in PBS and incubated for 8 hours at $37^{\circ} \mathrm{C}$ in staining solution containing $1 \mathrm{mg} / \mathrm{ml}$ 5 -bromo-4-chloro-3-indolyl- $\beta$-D-galactoside (X-Gal) (Roche) and the rest of the components described in [16]. For staining at different $\mathrm{pH}$ values, $0.1 \mathrm{M}$ citric acid and $0.2 \mathrm{M} \mathrm{Na}_{2} \mathrm{HPO}_{4}$ solutions were mixed at appropriate proportions. For $\mathrm{pH} 4.0-38.6 \mathrm{ml}$ of $0.2 \mathrm{M}$ $\mathrm{Na}_{2} \mathrm{HPO}_{4}$ were mixed with $61.5 \mathrm{ml}$ of $0.1 \mathrm{M}$ citric acid, and for $\mathrm{pH} 6.0-63.2 \mathrm{ml}$ of $0.2 \mathrm{M} \mathrm{Na}_{2} \mathrm{HPO}_{4}$ were mixed with $36.9 \mathrm{ml}$ of $0.1 \mathrm{M}$ citric acid. Following the incubation period at $37^{\circ} \mathrm{C}$, cells were washed $3 \times 5 \mathrm{~min}$ utes with cold PBS and stored in PBS at $4^{\circ} \mathrm{C}$ until images were collected.

For analysis of the effect of cell density on SABG activity, FSE cells from two different PDs (42 and 46) were seeded at increasing densities ranging from $20 \times$ $10^{3} /$ well to $250 \times 10^{3} /$ well (in 6 -well plates), and cultured until the well containing the highest cell density reached confluence.

\section{Diabetic animal model}

Two-month-old C57Bl/6 mice were injected intra-peritoneal for 5 consecutive days with the pancreatic islet $\beta$ cell toxin, STZ, at a concentration of $50 \mathrm{mg} / \mathrm{kg}$ dissolved in $50 \mathrm{mM}$ citrate buffer $\mathrm{pH} 4.5$. Blood glucose levels were monitored once a week with DM being defined as a consistent blood glucose level higher than $200 \mathrm{mg} / \mathrm{dl}$. Mice were sacrificed at 3 time points ( 7 weeks after injection, 9 weeks after injection and 13 weeks after injection). The experiment was repeated twice for the 9and 13-week time points. Anesthesia was carried out by injection of a mixture of ketamine and xylazine. Kidneys were removed and embedded in optimal cutting temperature compound (Tissue-Tek, Sakura), snap-frozen in liquid nitrogen-cooled methylbutane and stored at $-70^{\circ}$ C. The institutional animal care and welfare committee approved these experimental protocols.

\section{In-Situ SABG staining}

Frozen kidney sections were cut at $6 \mu \mathrm{m}$ and kept on dry ice until further processed. Slides were fixed with $2 \%$ formaldehyde $/ 0.2 \%$ glutaraldehyde in PBS for 5 minutes. After washing with ice cold PBS, slides were incubated over night at $37^{\circ} \mathrm{C}$ with SA- $\beta$-Gal staining solution previously described [16]. Following staining, slides were incubated for $48 \mathrm{~h}$ in ice cold PBS, and then were counterstained with eosin, dehydrated, and mounted as described [16].

\section{Microscopy, image analysis and BGAV analysis Analysis of tissue-culture cells}

Color images of SABG stained tissue culture cells were captured on a Nikon eclipse TS100 inverted microscope using a 10× objective and a Nikon Coolpix E995 digital camera 40-48 hours post staining. In several cases, specific wells were photographed several times at different intervals after staining was completed ( 8 hours to 2 months) to determine whether BGAVs change over time.

Quantitative analysis of the images was performed using a Matlab application for cell marking (SegmentGui) and color analysis (detailed instructions and download appear on website http://md.technion.ac.il/pictures/ storage/45/47.zip). A minimum of 250 randomly chosen cells was marked manually for each measurement point.

\section{Analysis of stained tissues}

The images of stained tissues were captured on a BX51 Olympus microscope using a $20 \times$ objective with an Olympus DP70 digital camera controlled by analySIS software (Soft Imaging System). Image acquisition was performed with the following fixed color settings: red = 0.36 , green $=0.92$, blue $=0.85$, offset $=504$, and exposure time of $6.8 \mathrm{~ms}$.

Analysis of BGAVs was performed on whole renal tubules (Figure 6). At least 50 tubules were analyzed from each mouse (DM and control) at each of the 3 time points. Experiments were carried out in duplicates for the DM mice at the 9- and 13-week time points.

\section{Statistical Analysis}

The distributions of SABG values were compared to a normal distribution by the use of Shapiro Wilk W normality test. Since most distributions deviated significantly from a normal distribution (see "Results"), we used the two sample Kolmogorov Smirnov test to calculate distribution differences between the different cell samples and for calculation of the $\mathrm{p}$ values.

\section{Immunofluorescence analysis of $\gamma-\mathrm{H} 2 \mathrm{AX}$ foci}

The staining of the mouse kidney tissue with antibodies for $\gamma$-H2AX was carried out as described previously [2]. Briefly, $8 \mu \mathrm{M}$ sections were cut from the frozen kidney biopsies and immediately fixed for 20 minutes in freshly prepared $4 \%$ paraformaldehyde diluted in PBS. Following the fixation, the tissue sections were permeabilized for 20 minutes with PBST (PBS + 0.2\% Triton X100). Sections were blocked for 1 hour in blocking solution consisting of $\mathrm{PBS}+4 \% \mathrm{BSA}$ at room temperature (RT) and incubated over night at $4{ }^{\circ} \mathrm{C}$ with anti- $\gamma-\mathrm{H} 2 \mathrm{AX}$ (S139, Upstate; Chicago, IL) diluted 1:750 in blocking solution. The following day, the slides were washed $3 \times$ 5 minutes in PBST at RT, and incubated with a secondary antibody (Cy3- conjugated donkey anti-rabbit antibody) for 1 hour at RT, Slides were then washed $3 \times 5$ minutes in PBST at RT and mounted with Vectashield containing DAPI (H-1200, VECTOR LABORATORIES). Fluorescence signals in stained tissue were analyzed using laser scanning confocal microscopy (LSM 510 
Meta Confocal, Carl Zeiss Inc. Germany). Nuclei were scored for the number of $\gamma$-H2AX foci present in each nucleus. Differences in number of foci between diabetic and control tissues were compared by Pearson's chisquare test.

\section{Acknowledgements}

We thank Maty Tzukerman for her helpful discussions and suggestions, Woodring Wright for providing us with BJ cells and Shiri Kalet-Litman for her help in the experiments with the diabetic mice. We are grateful to Sagi Abelson and Annie Rebibo-Sabbah for their technical help and to Ayala Ofir for her help with the confocal microscopy. This research was supported by the Israel Science Foundation (grant No. 542/05) (S.S.), the Deborah Grant (grant No 2007646) (K.S., L.S., S.S.), the Arthur and Rosalinde Gilbert Estate grants and the Sidney Kremer Kidney research Fund at the Technion (K.S).

\section{Author details}

'Laboratory of Molecular Medicine, Rappaport Faculty of Medicine and Research Institute, Technion, Haifa 31096, Israel. ${ }^{2}$ Israeli Naval Medical Institute, Haifa, Israel. ${ }^{3}$ Rambam Health Care Center, POB 9602, Haifa 31096, Israel. ${ }^{4}$ Department of Computer Science and Applied Mathematics, Weizmann Institute of Science, Rehovot 76100 Israel.

\section{Authors' contributions}

LIS conceived the study, participated in design of the study, carried out experiments, analyzed data, and participated in writing the manuscript. SI and $A C$ developed the software. AR, RB, SY and HS carried out the experiments and participated in analysis of the data. SS and KS conceived the study, participated in design of the study, and wrote the manuscript. All authors read and approved the final manuscript.

Received: 3 September 2010 Accepted: 15 April 2011

Published: 15 April 2011

\section{References}

1. Muller M: Cellular senescence: molecular mechanisms, in vivo significance, and redox considerations. Antioxid Redox Signal 2009, 11(1):59-98.

2. Herbig U, Ferreira M, Condel L, Carey D, Sedivy JM: Cellular senescence in aging primates. Science 2006, 311(5765):1257.

3. d'Adda di Fagagna F, Reaper PM, Clay-Farrace L, Fiegler H, Carr P, Von Zglinicki T, Saretzki G, Carter NP, Jackson SP: A DNA damage checkpoint response in telomere-initiated senescence. Nature 2003, 426(6963):194-198.

4. Gire V, Roux P, Wynford-Thomas D, Brondello JM, Dulic V: DNA damage checkpoint kinase Chk2 triggers replicative senescence. Embo J 2004, 23(13):2554-2563.

5. Campisi J, d'Adda di Fagagna F: Cellular senescence: when bad things happen to good cells. Nat Rev Mol Cell Biol 2007, 8(9):729-740.

6. Di Micco R, Fumagalli M, Cicalese A, Piccinin S, Gasparini P, Luise C, Schurra C, Garre M, Nuciforo PG, Bensimon A, Maestro R, Pelicci PG, d'Adda di Fagagna F: Oncogene-induced senescence is a DNA damage response triggered by DNA hyper-replication. Nature 2006, 444(7119):638-642.

7. Mallette FA, Gaumont-Leclerc MF, Ferbeyre G: The DNA damage signaling pathway is a critical mediator of oncogene-induced senescence. Genes Dev 2007, 21(1):43-48.

8. Bartkova J, Rezaei N, Liontos M, Karakaidos P, Kletsas D, Issaeva N, Vassiliou LV, Kolettas E, Niforou K, Zoumpourlis VC, Takaoka M, Nakagawa H, Tort F, Fugger K, Johansson F, Sehested M, Andersen CL, Dyrskjot L, Orntoft T, Lukas J: Oncogene-induced senescence is part of the tumorigenesis barrier imposed by DNA damage checkpoints. Nature 2006, 444(7119):633-637.

9. Lees-Miller SP: Dysfunction of lamin A triggers a DNA damage response and cellular senescence. DNA Repair (Amst) 2006, 5(2):286-289.

10. Von Kobbe C, May A, Grandori C, Bohr VA: Werner syndrome cells escape hydrogen peroxide-induced cell proliferation arrest. Faseb J 2004, 18(15):1970-1972.
11. Caldini R, Chevanne M, Mocali A, Tombaccini D, Paoletti F: Premature induction of aging in sublethally $\mathrm{H}_{2} \mathrm{O} 2$-treated young MRC5 fibroblasts correlates with increased glutathione peroxidase levels and resistance to DNA breakage. Mech Ageing Dev 1998, 105(1-2):137-150.

12. Toussaint O, Medrano EE, von Zglinicki T: Cellular and molecular mechanisms of stress-induced premature senescence (SIPS) of human diploid fibroblasts and melanocytes. Exp Gerontol 2000, 35(8):927-945.

13. Neumeister P, Albanese C, Balent B, Greally J, Pestell RG: Senescence and epigenetic dysregulation in cancer. Int J Biochem Cell Biol 2002, 34(11):1475-1490.

14. Campisi J: Senescent cells, tumor suppression, and organismal aging: good citizens, bad neighbors. Cell 2005, 120(4):513-522.

15. Campisi J: Suppressing cancer: the importance of being senescent. Science 2005, 309(5736):886-887.

16. Dimri GP, Lee X, Basile G, Acosta M, Scott G, Roskelley C, Medrano EE, Linskens M, Rubelj I, Pereira-Smith O, Peacocke M, Campisi J: A biomarker that identifies senescent human cells in culture and in aging skin in vivo. Proc Natl Acad Sci USA 1995, 92(20):9363-9367.

17. Itahana K, Campisi J, Dimri GP: Methods to detect biomarkers of cellular senescence: the senescence-associated beta-galactosidase assay. Methods Mol Biol 2007, 371:21-31.

18. Pendergrass WR, Lane MA, Bodkin NL, Hansen BC, Ingram DK, Roth GS, Yi L, Bin H, Wolf NS: Cellular proliferation potential during aging and caloric restriction in rhesus monkeys (Macaca mulatta). J Cell Physiol 1999, 180(1):123-130.

19. Michaloglou C, Vredeveld LC, Soengas MS, Denoyelle C, Kuilman T, van der Horst CM, Majoor DM, Shay JW, Mooi WJ, Peeper DS: BRAFE600-associated senescence-like cell cycle arrest of human naevi. Nature 2005, 436(7051):720-724

20. Braig M, Lee S, Loddenkemper C, Rudolph C, Peters AH, Schlegelberger B, Stein H, Dorken B, Jenuwein T, Schmitt CA: Oncogene-induced senescence as an initial barrier in lymphoma development. Nature 2005, 436(7051):660-665.

21. Gerland LM, Peyrol S, Lallemand C, Branche R, Magaud JP, Ffrench M: Association of increased autophasgic inclusions labeled for betagalactosidase with fibroblastic aging. Exp Gerontol 2003, 38(8):887-895.

22. Kurz DJ, Decary S, Hong Y, Erusalimsky JD: Senescence-associated (beta)galactosidase reflects an increase in lysosmal mass during replicative ageing of human endothelial cells. J Cell Sci 2000, 113:3613-3622.

23. Lee BY, Han JA, Im JS, Morrone A, Johung K, Goodwin EC, Kleijer WJ, DiMaio D, Hwang ES: Senescence-associated beta-galactosidase is lysosomal beta-galactosidase. Aging Cell 2006, 5(2):187-195.

24. Yegorov YE, Akimov SS, Hass R, Zelenin AV, Prudovsky IA: Endogenous beta-galactosidase activity in continuously nonproliferating cells. Exp Cell Res 1998, 243(1):207-211

25. Severino J, Allen RG, Balin S, Balin A, Cristofalo VJ: Is beta-galactosidase staining a marker of senescence in vitro and in vivo? Exp Cell Res 2000, 257(1):162-171.

26. Yang NC, Hu ML: The limitations and validities of senescence associatedbeta-galactosidase activity as an aging marker for human foreskin fibroblast Hs68 cells. Exp Gerontol 2005, 40(10):813-819.

27. Toussaint O, Dumont P, Remacle J, Dierick JF, Pascal T, Frippiat C, Magalhaes JP, Zdanov S, Chainiaux F: Stress-induced premature senescence or stress-induced senescence-like phenotype: one in vivo reality, two possible definitions? ScientificWorldJournal 2002, 2:230-247.

28. Coelho JC, Sopelsa AM, Tobo PR, Severini MH, Silva CD, Giugliani R: Biochemical studies on leukocyte and fibroblast human betagalactosidase. Clin Biochem 1999, 32(3):167-170.

29. Sis B, Tasanarong A, Khoshjou F, Dadras F, Solez K, Halloran PF: Accelerated expression of senescence associated cell cycle inhibitor p16INK4A in kidneys with glomerular disease. Kidney Int 2007, 71(3):218-226.

30. Jimenez R, Carracedo J, Santamaria R, Soriano S, Madueno JA, Ramirez R, Rodriguez M, Martin-Malo A, Aljama P: Replicative senescence in patients with chronic kidney failure. Kidney Int Supp/ 2005, , 99: S11-15.

31. Melk A, Schmidt BM, Takeuchi O, Sawitzki B, Rayner DC, Halloran PF: Expression of p16INK4a and other cell cycle regulator and senescence associated genes in aging human kidney. Kidney Int 2004, 65(2):510-520.

32. Verzola D, Gandolfo MT, Gaetani G, Ferraris A, Mangerini R, Ferrario F, Villaggio B, Gianiorio F, Tosetti F, Weiss U, Traverso P, Mji M, Deferrari G, Garibotto G: Accelerated senescence in the kidneys of patients with type 
2 diabetic nephropathy. Am J Physiol Renal Physiol 2008, 295(5):

F1563-1573.

33. Asaf R, Blum S, Roguin A, Kalet-Litman S, Kheir J, Frisch A, Miller-Lotan R,

Levy AP: Haptoglobin genotype is a determinant of survival and cardiac remodeling after myocardial infarction in diabetic mice. Cardiovasc Diabetol 2009, 8:29.

34. Wang C, Jurk D, Maddick M, Nelson G, Martin-Ruiz C, von Zglinicki T: DNA damage response and cellular senescence in tissues of aging mice. Aging Cell 2009, 8(3):311-323.

35. Cristofalo VJ: SA beta Gal staining: biomarker or delusion. Exp Gerontol 2005, 40(10):836-838

36. Gary RK, Kindell SM: Quantitative assay of senescence-associated betagalactosidase activity in mammalian cell extracts. Anal Biochem 2005, 343(2):329-334.

37. Yang NC, Hu ML: A fluorimetric method using fluorescein di-beta-Dgalactopyranoside for quantifying the senescence-associated betagalactosidase activity in human foreskin fibroblast $\mathrm{Hs} 68$ cells. Anal Biochem 2004, 325(2):337-343.

38. Bassaneze V, Miyakawa AA, Krieger JE: A quantitative chemiluminescent method for studying replicative and stress-induced premature senescence in cell cultures. Anal Biochem 2008, 372(2):198-203.

39. Noppe G, Dekker P, de Koning-Treurniet C, Blom J, van Heemst D, Dirks RW, Tanke HJ, Westendorp RG, Maier AB: Rapid flow cytometric method for measuring senescence associated beta-galactosidase activity in human fibroblasts. Cytometry A 2009, 75(11):910-916.

40. Krishna DR, Sperker B, Fritz P, Klotz U: Does pH 6 beta-galactosidase activity indicate cell senescence? Mech Ageing Dev 1999, 109(2):113-123.

41. Yalon M, Gal S, Segev Y, Selig S, Skorecki KL: Sister chromatid separation at human telomeric regions. J Cell Sci 2004, 117(Pt 10):1961-1970.

42. Ouellette MM, Aisner DL, Savre-Train I, Wright WE, Shay JW: Telomerase activity does not always imply telomere maintenance. Biochem Biophys Res Commun 1999, 254(3):795-803.

doi:10.1186/1471-2121-12-16

Cite this article as: Shlush et al:: Quantitative digital in situ senescenceassociated $\beta$-galactosidase assay. BMC Cell Biology 2011 12:16.

\section{Submit your next manuscript to BioMed Central and take full advantage of:}

- Convenient online submission

- Thorough peer review

- No space constraints or color figure charges

- Immediate publication on acceptance

- Inclusion in PubMed, CAS, Scopus and Google Scholar

- Research which is freely available for redistribution

Submit your manuscript at www.biomedcentral.com/submit 